\title{
JEAN-RODOLPHE PERRONET
}

Premier Ingénieur des Ponts et Chaussées de France

Fondateur et premier directeur de l'Ecole Nationale des Ponts et Chaussées

$1708-1794$

On a fêté, récemment, le bi-centenaire de l'Ecole Nationale des Ponts et Chaussées et, à cette occasion, la vie et l'œuvre de PERRONET ont bénéficié d'un certain regain d'actualité.

Nous signalerons, entre autres, la très intéressante étude de M. H. MarCEL, Ingénieur des Ponts et Chausisées, étude intitulêe: "Les débuts de J.-R. PERronet, Ingénieur des Ponts et Chaussées de la généralité d'Alençon (1). »

Récemment, nous tombions, par hasard, sur un exemplaire de l'édition originale de la biographie de PERronet, par Lesage.

Cet exemplaire porte, sur le's dernières pages, des noteis manuscrites dictées en 1804 par l'Abbé Goy, de Grenoble, ami intime de Perroner, précisant certains aspects de la personnalité du premier Ingénieur des Pionts et Chaussées de France.

Nous reproduisons, ci-dessous, quelques extraits de ces notes; nous iserions heureux d'avoir l'avis de nos lecteurs sur l'intérêt que présenterait une rubrique de ce genre, consacrée aux personnages célèbres de l'histoire de l'Hydraulique.

Jean-Rodolphe PERRONET naquit à Suresnes près Paris, le 8 octobre 1708. Son père était officier suisse. Originaire du canton de Berne, parent de $M$. DE CROUZAN, l'un des savants les plus illustres de notre siècle ............

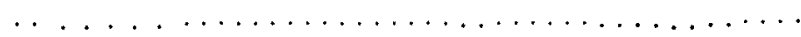

dès sa plus tendre jeunesse, ...........

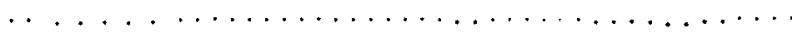
il s'appliqua avec ardeur à toutes les études qu'on voulut lui faire suivre.

un livre de physique qu'il trouva par hasard parmi ceux de son père, attira son attention et saisit bientôt son cœur. II parcourut avidement cet ouvrage ; malheureusement, n'ayant aucune des

(1) Annales des Ponts et Chaussées, juillet-août 1948, page 419 (numéro 26). notions de calcul et de géométrie que l'auteur supposait, il se trouvait arrêté à chaque pas, et ne faisait qu'entrevoir la vérité qu'il brûlait de connaître. Il eût bien désiré de savoir un peu de cette géométrie dont la physique emprunte tant de secours, mais pour cela, il fallait un maître que ses parents n'étaient guère en état de payer.

Cependant, ils consentirent à le laisser suivre un cours public et gratuit de mathématiques qui se faisait au collège de Grassins.

Dès ce moment, il ne connut plus d'autre plaisir que l'étude; il y employait des journées entières, il y passait quelques fois des nuits, et sa sonté en fut souvent altérée. Ses progrès furent tels, qu'avant l'âge de 16 ans il se trouva en état d'aspirer à une place dans le corps des ingénieurs militaires.

En 1745, il quitta cette place (auprès de $M$. BEAUSIRE, architecte de la ville de Paris), pour entrer dans les Ponts et Chaussées ; il fut nommé ingénieur en chef de la généralité d'Alençon, et peu de temps après appelé à Paris pour être directeur général.

Jusqu'à cette époque, les ingénieurs des Ponts et Chaussées ne formaient point un corps, et on les connaissait à peine ; la plupart avaient été choisis parmi de simples architectes. Un très petit nombre d'entre eux s'étaient distingués il est vrai, par leur talent ; mais les autres n'étaient proprement dit que des constructeurs sans théorie et sans principe, et les ingénieurs en second en savaient moins que les piqueurs qu'on emploie aujourd'hui.

M. DE TRUDAINE ayant pris ce département dont il connut le premier toute l'importance, sentit qu'il était absolument nécessaire d'établir à Paris une école où seraient instruits, par d'excellents maîtres, et sévèrement examinés sur toutes les parties de l'art, les sujets qui se

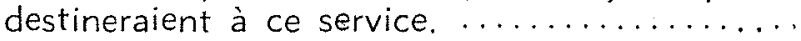




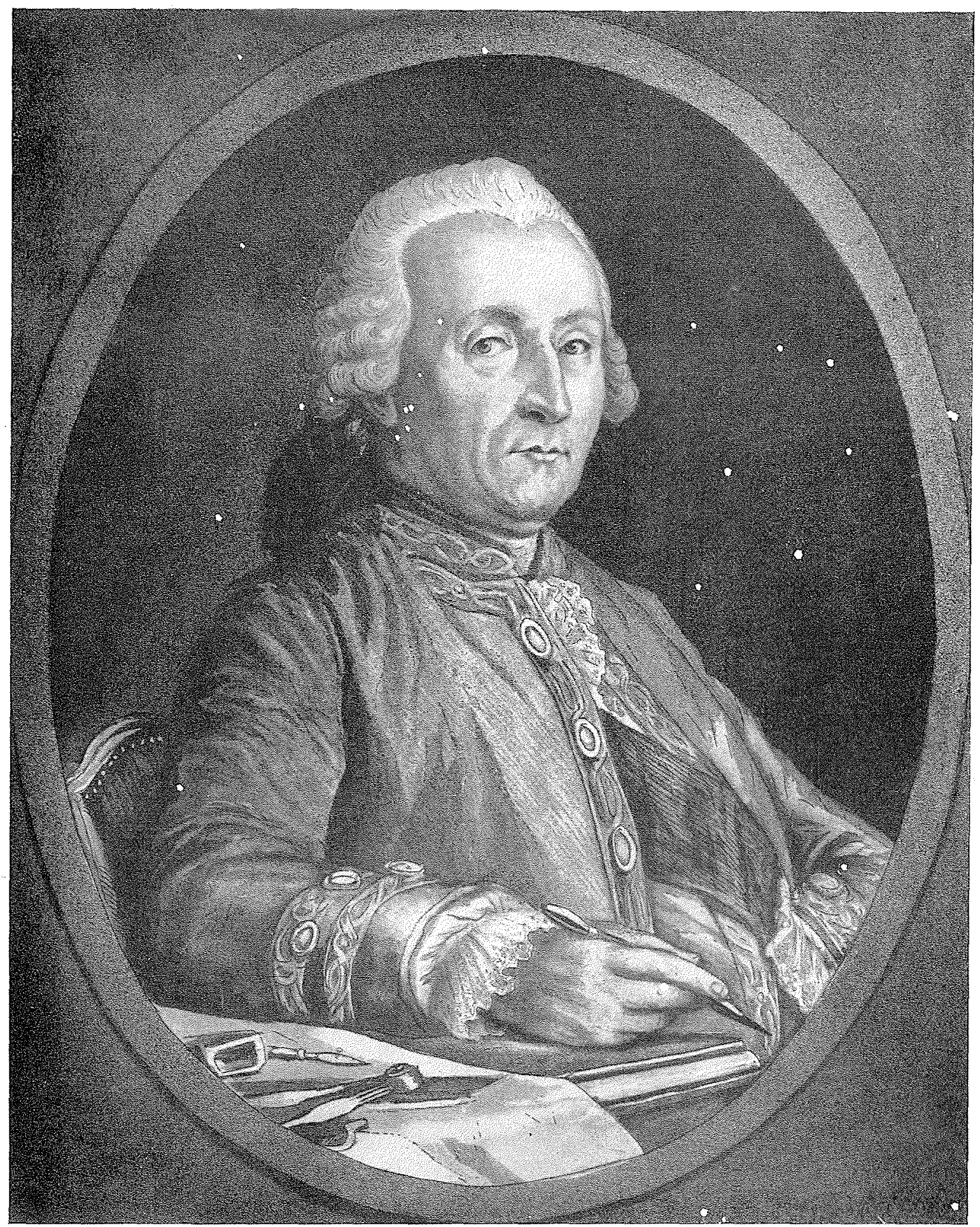

Cochin filties Del.

Queneday Sonts.

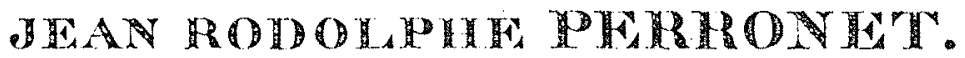

CIIFAJIH'R DE L'ORDRE DE ST AITHEL.

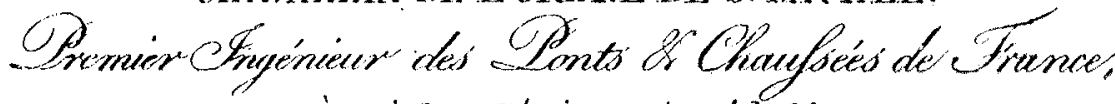

Mort à laris, le a7. Février 1794 . agé de 86 ans. 
Après avoir médité son dessein, il voulut avant d'en venir à l'exécution, consulter M. PERRONET qu'il avait jugé propre à seconder ses vues, et il crut ne pouvoir mieux faire que de lui confier la direction du nouvel Etablissement.

Il eut tout lieu de s'applaudir de son choix. Bientôt, au sentiment d'estime qui l'avait déterminé se joignit celui d'une amitié particulière, que le temps devait fortifier de plus en plus et qui ne s'est jamais démentie.

Ces deux hommes étaient faits pour se convenir parfaitement : l'un administrateur très éclairé, excellent citoyen, ami de l'ordre et de l'économie, d'une intégrité et d'une fermeté qui le rendaient inaccessible à toute sollicitation; l'autre, profond dans son art, laborieux, désintéressé, incapable de sacrifier aucun de ses devoirs à ses affections personnelles. Il résulta de cet accord tout le bien qu'on s'était promis d'une institution aussi bien conçue. Elle se consolida par les plus sages règlements, par la vigilance et l'équité de ses directeurs et par le mérite d'un grand nombre de sujets qui y furent élevés.

Le Gouvernement et le public en ont si bien reconnu l'utilité que, pendant près de 50 ans, le régime qui y fut établi dans le principe, n'a souffert aucune altération. Ce fait peut surprendre quand on a vu sous les deux derniers règnes, les ministres se succéder avec une telle rapidité, qu'à peine a-t-on pu retenir leur nom, et chacun d'eux absolu dans son département, débutait toujours par détruire, bien ou mal construit, l'édifice

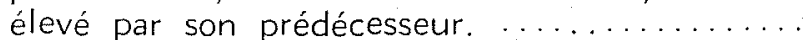

C'est du moment où M. PERRONET arriva à la tête des Ponts et Chaussées, que commença la réputation de ce corps qui s'est illustré par un très grand nombre d'hommes à talent, formés à son école, où l'on a vu plusieurs puissances étrangères envoyer leurs ingénieurs pour y perfectionner leur instruction.

Il semblerait qu'avec cet esprit de modération et de désintéressement, il ne devait point avoir d'ennemis, même parmi ses rivaux. Il en eut cependant que sa supériorité gênait et chagrinait fort, mais au lieu de s'en offenser et de se plaindre, soit de leur censure, soit de leur procédé, on l'a vu plus d'une fois servir généreusement et à leur insu leurs prétentions par le sacrifice des siennes. L'on peut assurer que sa bonté naturelle et non sa politique se plaisait à désarmer ainsi leur jalousie qu'il eut pu mépriser.

Il avait été marié à une femme qui ne le rendit pas heureux et dont il n'eut point d'enfant ; mais il se composa par la suite une famille nombreuse de tous les élèves de son école qui devinrent l'objet principal de son affection et de ses soins.

Pendant près de 50 ans il présida à cette école et quelque temps avant sa mort, il a vu le corps des ingénieurs renouvelé en entier, et toutes les places sans exception successivement remplies par ses élèves.

Il jouissait d'un traitement assez considérable, plus que suffisant pour ses désirs, et qu'il ne fut jamais tenté d'employer en dépenses fastueuses. Sa maison était entretenue honnêtement, mais très simplement, et le reste de ses revenus était destiné à acquitter des pensions qu'il faisait à plusieurs de ses parents, à soulager beaucoup de malheureux qui avaient recours à sa charité, à en prévenir d'autres qui n'eussent osé venir lui exposer leurs besoins, à fournir des secours à des jeunes gens qui n'étaient pas en état de payer les frais de leur instruction, ....

A 86 ans, il avait conservé avec le jugement le plus sain, une extrême sensibilité, un courage que rien n'étonnait, une patience à toute épreuve, une candeur et une aménité qui faisaient le bonheur et l'admiration de tous ceux qui vivaient avec lui dans un commerce intime.

II était associé libre de l'Académie des Sciences de Paris, membre de celles de Berlin et de Stockholm, et de la Société Royale de Londres qui dérogea en sa faveur à un arrêté qu'elle avait pris pour n'admettre dans son sein, aucun associé étranger dont elle voulait réduire considérablement le nombre. Il avait été nommé plus de 30 ans avant sa mort, Chevalier dé l'Ordre du Roi.

Tous ces honneurs furent le prix de ses services et de ses talents. L'estime des hommes qui l'ont connu, la reconnaissance de ceux à qui il a fait du bien, les regrets éternels de ses amis, et leur respect pour sa mémoire, sont la récompense acquise à ses vertus. 